\title{
O ESTRESSE NO MMA: AS ESTRATÉGIAS DE \\ ENFRENTAMENTO PODEM MELHORAR O DESEMPENHO DOS LUTADORES?
}

\author{
STRESS IN MMA: CAN COPING STRATEGIES IMPROVE THE PERFORMANCE OF FIGHTERS?
}

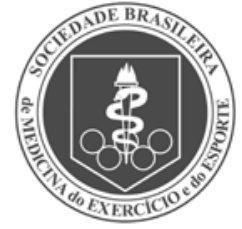

Artigo Original

Original ARTICLE

Artículo Original

\section{ESTRÉS EN MMA: ¿LAS ESTRATEGIAS DE ENFRENTAMIENTO PUEDEN MEJORAR EL RENDIMIENTO DE LOS LUCHADORES?}

Isabella Belem¹ (Educadora Física) Luciane Cristina Arantes da Costa ${ }^{2}$ (Educadora Física)

Jorge Both ${ }^{3}$ (Educador Físico)

Patricia Carolina Borsato Passos ${ }^{2}$ (Educadora Física)

José Luiz Lopes Vieira²

(Educador Físico)

1. Universidade Paranaense (Unipar), Maringá, PR, Brasil. 2. Universidade Estadual de Maringá (UEM), Maringá, PR, Brasil. 3. Universidade Estadual de Londrina (UEL), Londrina, PR, Brasil.

\section{Correspondência:}

Luciane Cristina Arantes da Costa Departamento de Educação Física Av. Colombo, 5790, Jardim Universitário, Maringá, PR, Brasil. 87020-900.

luarantes100@gmail.com

\section{RESUMO}

Introdução: Um dos maiores fenômenos esportivos na atualidade, o Ultimate Fighting Championship (UFC), realizado no Brasil em 2013, movimentou entre R\$ 15 e R\$ 50 milhões, destacando-se na mídia internacional. Apesar dessa superprodução dos eventos, os estudos com atletas de MMA (Mixed Martial Arts) ainda são escassos, sobretudo no que se refere aos aspectos psicológicos. Objetivo: Analisar estratégias de coping, níveis de estresse e características de treinamento de atletas paranaenses de MMA. Métodos: Foram estudados 50 atletas de MMA do sexo masculino, com idade de 25,0 \pm 4,8 anos. Como instrumentos foram utilizados uma ficha de identificação, o Inventário Atlético de Estratégias de Coping e o Questionário de Estresse e Recuperação para Atletas (RESTQ-76 Sport). Na análise dos dados, utilizou-se estatística descritiva, teste de Shapiro-Wilk, teste "U" de Mann-Whitnney; teste de Kruskal-Wallis e coeficiente de correlação de Spearman; a significância adotada foi $p<0,05$. Resultados: Os atletas com principal fonte de renda no MMA apresentaram maior "estresse geral" $(p=0,023)$ e "estresse social" ( $p=0,043)$. Conclusão: Conclui-se que quando o esporte é a principal fonte de renda dos atletas, o estresse dos lutadores é elevado. Quanto maior o volume de treino, mais o atleta busca estabelecer objetivos e maior é sua a capacidade de confrontar as adversidades. Além disso, a variedade de estratégias de coping utilizadas pelos lutadores melhora seu estado de recuperação (capacidades físicas e psicológicas) durante as competições.

Descritores: atletas; esgotamento profissional; esportes.

\section{ABSTRACT}

Introduction: One of the biggest sporting phenomena today, the Ultimate Fighting Championship (UFC), held in Brazil in 2013, had a turnover of between $R \$ 5$ and $R \$ 15$ million, with extensive coverage in the international media. Despite this high production of events, studies with MMA (Mixed Martial Arts) athletes are still rare, particularly concerning psychological aspects. Objective: To analyze coping strategies, stress levels, and training characteristics

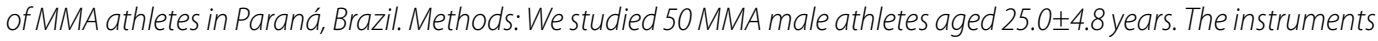
used were an identification form, the Athletic Coping Skills Inventory and the Recovery-Stress Questionnaire for Athletes (RESTQ-76 Sport). For the data analysis, we used descriptive statistics, the Shapiro-Wilk test, the Mann-Whitney "U" test, the Kruskal-Wallis test and Spearman's rank correlation coefficient; a level of significance $p<0.05$ was adopted. Results: Athletes whose main source of income is MMA had higher "general stress" ( $p=0.023$ ) and "social stress" ( $p=0.043)$. Conclusion: We conclude that when the sport is the main athletes' source of income, the stress of the fighters is high. The higher the volume of training, the more the athlete seeks to set goals and the greater his or her ability to confront adversity. Moreover, the variety of coping strategies used by the fighters improves their state of recovery (physical and psychological capacities) during competitions.

Keywords: athletes; burnout, professional; sports.

\section{RESUMEN}

Introducción: Uno de los mayores fenómenos deportivos de la actualidad, el Ultimate Fighting Championship (UFC), celebrado en Brasil en 2013, movilizó entre $R \$ 15$ y $R \$ 50$ millones, destacándose en los medios de comunicación internacionales. A pesar de esta gran producción de eventos, los estudios con atletas de MMA (Mixed Martial Arts) son todavía escasos, sobre todo en lo que respecta a los aspectos psicológicos. Objetivo: El analizar las estrategias de enfrentamiento, los niveles de estrés y las características de entrenamiento de atletas de MMA del Paraná, Brasil. Métodos: Se estudiaron 50 atletas de MMA del sexo masculino, con edades de 25,0 \pm 4,8 años. Como instrumentos se utilizaron una ficha de identificación, el Inventario de Habilidades Deportivas de Afrontamiento y el Cuestionario de Recuperación-estrés para Deportistas (RESTQ-76 Sport). En el análisis de los datos se utilizó la estadística descriptiva, prueba de Shapiro-Wilk, prueba de "U" de Mann-Whitnney; prueba de Kruskal-Wallis y el coeficiente de correlación de Spearman; la significación adoptada fue de $p<0,05$. Resultados: Los atletas que tienen su principal fuente de ingresos en MMA tuvieron "estrés en general" ( $p=0,023)$ y "estrés social" $(p=0,043)$ superiores. 
Conclusión: Se concluye que cuando el deporte es la principal fuente de ingresos de los atletas el estado de estrés de los luchadores es alto. Cuanto mayor sea el volumen de entrenamiento, más el atleta busca establecer las metas y mayor será su capacidad para hacer frente a la adversidad. Además, la variedad de estrategias de enfrentamiento utilizadas por los luchadores mejora su estado de recuperación (capacidades físicas y psicológicas) durante las competiciones.

Descriptores: atletas; agotamiento profesional; deportes.

\section{INTRODUÇÃO}

A partir da criação do Ultimate Fighting Championship (UFC), no ano de 1993 nos Estados Unidos, aumentou o interesse dos fãs e meios de comunicação, e consequentemente o número de praticantes de Mixed Martial Arts (MMA) ${ }^{1,2}$. Nos últimos anos no Brasil, a procura por este esporte aumentou cerca de 100\% nas academias. Além disso, a última edição do UFC, realizada no país, movimentou entre $\mathrm{R} \$ 15 \mathrm{e}$ $\mathrm{R} \$ 50$ milhões e seus impactos se estendem por diversos setores como, por exemplo, o turismo. Outro fator que pode estar ligado ao aumento da procura pelo esporte é o incentivo financeiro para aqueles que competem em alto nível ${ }^{3}$.

Os estudos publicados com atletas de MMA buscaram investigar a quantidade e a gravidade de lesões sofridas ${ }^{2,4,5}$, métodos de treinamento ${ }^{6-8}$ atratividade e agressão ${ }^{9}$, perfil fisiológico de lutadores ${ }^{10}$, aprendizagem e integração de competências técnicas ${ }^{11}$ e demandas psicológicas de atletas dessa modalidade ${ }^{3}$. Assim com a popularização da modalidade, os estudos que investigam os atletas de MMA têm crescido, no entanto, ainda há lacuna na literatura sobre como os fatores psicológicos podem auxiliar os lutadores a obter um meIhor desempenho.

Dentre as capacidades psicológicas dos atletas, as estratégias de enfrentamento de estresse (coping), têm sido descritas como um diferencial entre atletas de sucesso de diversas modalidades ${ }^{12,13}$. As estratégias de coping podem ser definidas como um conjunto de esforços cognitivos e comportamentais utilizados para administrar demandas internas ou externas que são avaliadas pela pessoa como estressantes ${ }^{14}$. Portanto, é de suma importância que os atletas de MMA se utilizem das estratégias de enfrentamento (coping) para superar fatores estressantes e manter bom desempenho, adaptando-se às mudanças nas demandas contextuais ${ }^{15}$. Contudo, a incapacidade na utilização dessas estratégias para lidar com o estresse pode afetar de forma negativa o desempenho do atleta, levando-o a falhas de atuação e derrotas ${ }^{16}$.

O esporte de alto rendimento tem sido apontado como um ambiente estressante para os atletas ${ }^{17-19}$ devido à necessidade de desempenho e sucesso ${ }^{20}$. Altos níveis de estresse podem causar lesões e doenças ${ }^{21}$ e dificultam a recuperação dos atletas após treinamentos intensos ${ }^{22}$, e na participação em competições ${ }^{23}$.

Desta forma, buscando preencher a lacuna identificada na literatura, este estudo teve como objetivo analisar as estratégias de coping, estado de estresse e características de treinamento de atletas paranaenses de MMA.

\section{MATERIAIS E MÉTODOS}

Participaram do estudo 50 atletas paranaenses de MMA do sexo masculino, com média de idade de 25,0 $\pm 4,8$ anos. Os atletas praticam a modalidade há 7,8 $\pm 4,7$ anos. O tempo médio de treino diário foi de $4,4 \pm 1,7$ horas, com média semanal de 23,0 \pm 9,7 horas e participam em competições há 5,2 \pm 3,9 anos, sendo que no momento atual 35 são de nível nacional e 15 de nível internacional. Os atletas participantes do estudo encontravam-se em período de preparação geral $(n=19)$ e no período pré-competitivo $(n=31)$.

\section{Instrumentos}

As variáveis relacionadas ao treinamento dos atletas foram identificadas por meio de uma ficha contendo questões relacionadas à: idade, estado civil, categoria em que luta, tempo de prática, tempo de treino diário, tempo de treino semanal, tempo de participação em competições, período de treinamento, nível de competição, remuneração pelas lutas, MMA como principal fonte de renda.

Para a investigação das estratégias de coping, foi utilizado o Inventário Atlético de Estratégias de Coping (ACSI-28) desenvolvido por Smith et al. ${ }^{24}$, validado para a língua portuguesa por Serpa e Palmeira ${ }^{25}$, composto por 28 itens em uma escala Likert de 4 pontos, "quase nunca" até "quase sempre". Os resultados são classificados em 7 subescalas: rendimento máximo sob pressão, ausência de preocupações, confronto com a adversidade, concentração, formulação de objetivos, confiança e motivação para a realização e treinabilidade. A média de todas as subescalas do instrumento indica o Índice de Confronto Geral no Esporte.

Para avaliar o estado de estresse, utilizou-se o Questionário de Estresse e Recuperação para Atletas (RESTQ-76 Sport) desenvolvido por Kellmann et al. ${ }^{26}$ e validado por Costa e Samulski ${ }^{27}$. A consistência interna do instrumento foi entre $a=0,58$ e $a=0,85$, indicando uma confiabilidade moderada aceitável de acordo com as recomendações psicométricas ${ }^{28}$. O questionário compreende 77 itens, sendo um introdutório (item número 1), que não está incluído no escore final. Cada subescala do RESTQ-76 Sport consiste em quatro itens avaliados por uma escala Likert com valores que variam de 0 a 6 pontos ( $0=$ nunca a $6=$ sempre), indicando a incidência de eventos e atividades relatadas.

\section{Procedimentos}

Após a autorização da Federação Paranaense de MMA, o projeto foi submetido e aprovado pelo Comitê Permanente de Ética em Pesquisa com Seres Humanos da Universidade Estadual de Maringá (Protocolo nº 267.975/2013). As coletas ocorreram nos locais de treino mediante agendamento prévio com os técnicos e os atletas, bem como assinatura de todos os participantes do Termo de Consentimento Livre e Esclarecido. A aplicação dos instrumentos foi realizada pela pesquisadora responsável de forma individual, tendo duração média de 30 minutos por atleta.

\section{Análise dos dados}

Para análise da distribuição dos dados foi utilizado o teste Shapiro-Wilk. Como os dados não apresentaram distribuição normal utilizou-se Mediana (Md), Quartis (Q1; Q3), frequência relativa e absoluta. Para a comparação entre os grupos (esporte como principal fonte de renda, período de treino) utilizou-se o teste "Prova "U" de Mann-Whitnney. O Teste Qui-quadrado foi utilizado para a associação entre nível de competição e características de treinamento. O nível de significância no estudo foi de $95 \%(p \leq 0,05)$.

\section{RESULTADOS}

A maioria dos atletas participa de competições na categoria de peso meio-médio (22\%), seguido da categoria de peso pena (18\%), meio pesado (16\%), leve (14\%), galo (12\%), mosca (6\%), médio (6\%) e pesado (6\%). A participação em competições internacionais associou-se ao maior 
tempo de prática $(p=0,019)$, ser remunerado pelas lutas $(p=0,034)$ e ter o MMA como principal fonte de renda (Tabela 1).

As comparações entre o estado de estresse e a questão referente à renda mensal obtida pelo esporte demonstraram que para os lutadores em que a modalidade é a principal fonte de renda, a ocorrência do estado de "estresse geral" ( $p=0,023)$ e "estresse social" $(p=0,043)$ foram maiores do que para os atletas que não dependem financeiramente do esporte. Não foram encontradas diferenças significativas para as demais categorias de estresse e estratégias de coping. Também não foram encontradas diferenças entre remuneração pelas lutas, estresse e estratégias de coping (Tabela 2).

Quanto ao período de treino, os lutadores que se encontravam no período pré-competitivo apresentaram maior "autorregulação" $(p=0,047)$ quando comparados aos do período de preparação geral. Não foram encontradas diferenças significativas para as demais categorias de estresse e estratégias de coping (Tabela 3).

A correlação entre as estratégias de coping e os dados referentes ao treinamento dos lutadores de MMA demonstrou que quanto maior o tempo de treino diário maior o "Índice de confronto geral" dos atletas $(r=0,31 ; p=0,03)$. Um maior volume de treino semanal está relacionado à "ausência de preocupações" ( $r=0,29 ; p=0,02)$, "formulação de objetivos" $(r=0,33 ; p=0,04)$ e ao "índice de confronto geral" $(r=0,35 ; p=0,01)$. A participação em competições está relacionada a um maior "confronto com as adversidades" ( $r=0,30 ; p=0,047$ ) (Tabela 4).

Tabela 1. Associação entre nível de competição (nacional e internacional) e características de treinamento de lutadores de MMA.

\begin{tabular}{|c|c|c|c|c|c|c|}
\hline \multirow{3}{*}{\multicolumn{2}{|c|}{ Características de treinamento }} & \multicolumn{4}{|c|}{ Nível de competição } & \multirow{3}{*}{$p^{*}$} \\
\hline & & \multicolumn{2}{|c|}{$\begin{array}{l}\text { Nacional } \\
(n=35)\end{array}$} & \multicolumn{2}{|c|}{$\begin{array}{l}\text { Internacional } \\
\qquad(n=15)\end{array}$} & \\
\hline & & $f$ & $\%$ & $f$ & $\%$ & \\
\hline \multirow{2}{*}{ Tempo de prática } & Até 5 anos & 17 & 48,6 & 02 & 13,3 & \multirow{2}{*}{0,019} \\
\hline & $\geq 6$ anos & 18 & 51,4 & 13 & 86,7 & \\
\hline \multirow{2}{*}{ Treino diário } & Até 4hs & 19 & 54,3 & 05 & 33,3 & \multirow{2}{*}{$0,174^{*}$} \\
\hline & $\geq 5 \mathrm{hs}$ & 16 & 45,7 & 10 & 66,7 & \\
\hline \multirow{2}{*}{ Treino semanal } & Até 20hs & 19 & 54,3 & 06 & 40,0 & \multirow{2}{*}{$0,355^{*}$} \\
\hline & $\geq 21 \mathrm{hs}$ & 16 & 45,7 & 09 & 60,0 & \\
\hline \multirow{2}{*}{ Período de treinamento } & Geral & 21 & 60,0 & 10 & 33,3 & \multirow{2}{*}{$0,565^{*}$} \\
\hline & Pré-competitivo & 14 & 40,0 & 05 & 66,7 & \\
\hline \multirow{2}{*}{ Remuneração pelas lutas } & Sim & 24 & 75,0 & 15 & 100,0 & \multirow{2}{*}{0,034} \\
\hline & Não & 08 & 25,0 & - & - & \\
\hline \multirow{2}{*}{$\begin{array}{l}\text { MMA como principal } \\
\text { fonte de renda }\end{array}$} & $\operatorname{sim}$ & 11 & 42,3 & 12 & 85,7 & \multirow{2}{*}{0,008} \\
\hline & Não & 15 & 57,7 & 2 & 14,3 & \\
\hline
\end{tabular}

* Probabilidade estimada pelo este qui-quadrado, diferença significativa $(p<0,05)$.

Tabela 2. Comparação entre os lutadores de MMA cuja prática esportiva é a principal fonte de renda e estado de estresse $(n=50)$.

\begin{tabular}{|c|c|c|c|c|c|}
\hline \multirow{3}{*}{ Estresse Geral } & \multicolumn{4}{|c|}{ MMA principal fonte de renda } & \multirow{3}{*}{$p^{*}$} \\
\hline & \multicolumn{2}{|c|}{$\operatorname{Sim}(n=19)$} & \multicolumn{2}{|c|}{ Não (n=31) } & \\
\hline & Md & (Q1-Q3) & Md & (Q1-Q3) & \\
\hline Estresse geral & 1,50 & $(0,62-2,41)$ & 0,75 & $(1,00-1,50)$ & 0,023 \\
\hline Estresse emocional & 1,50 & $(1,25-2,62)$ & 1,62 & $(1,00-2,25)$ & $0,401^{*}$ \\
\hline Estresse social & 1,25 & $(1,00-2,62)$ & 0,62 & $(0,50-1,50)$ & 0,043 \\
\hline Conflitos / Pressão & 2,75 & $(2,25-3,75)$ & 2,37 & $(2,00-3,37)$ & $0,072^{*}$ \\
\hline Fadiga & 2,50 & $(1,62-3,54)$ & 2,12 & $(0,87-3,12)$ & $0,150^{*}$ \\
\hline Falta de energia & 1,75 & $(0,87-2,25)$ & 1,25 & $(0,62-1,87)$ & $0,178^{*}$ \\
\hline Queixas somáticas & 1,75 & $(1,25-2,75)$ & 1,75 & $(0,71-2,50)$ & $0,415^{*}$ \\
\hline
\end{tabular}

Tabela 3. Comparação entre estado de recuperação e período de treino de lutadores de MMA.

\begin{tabular}{|c|c|c|c|c|c|}
\hline \multirow{3}{*}{ Recuperação Específica } & \multicolumn{4}{|c|}{ Período de treino } & \multirow{3}{*}{$p^{*}$} \\
\hline & \multicolumn{2}{|c|}{$\begin{array}{l}\text { Preparação geral } \\
\qquad(n=19)\end{array}$} & \multicolumn{2}{|c|}{$\begin{array}{l}\text { Pré-competitivo } \\
\qquad(n=31)\end{array}$} & \\
\hline & Md & (Q1-Q3) & Md & (Q1-Q3) & \\
\hline Estar em forma & 4,25 & $(3,50-4,75)$ & 4,75 & $(4,00-5,75)$ & $0,073^{*}$ \\
\hline Aceitação Pessoal & 4,50 & $(3,54-5,12)$ & 4,75 & $(4,00-5,75)$ & $0,237^{*}$ \\
\hline Autoeficácia & 4,25 & $(3,87-5,37)$ & 5,00 & $(4,12-5,50)$ & $0,341^{*}$ \\
\hline Autorregulação & 5,00 & $(3,62-5,50)$ & 5,50 & $(4,87-5,75)$ & 0,047 \\
\hline
\end{tabular}

* Probabilidade estimada pelo teste Prova $U$ de Mann-Whitney.

Tabela 4. Correlação entre estratégias de coping e características de treinamento de lutadores paranaenses de MMA $(n=50)$.

\begin{tabular}{c|c|c|c|c}
\hline \multirow{2}{*}{ Estratégias de Coping } & \multicolumn{4}{|c}{ Características de Treinamento } \\
\cline { 2 - 5 } & $\begin{array}{c}\text { Tempo de } \\
\text { prática }\end{array}$ & $\begin{array}{c}\text { Treino } \\
\text { diário }\end{array}$ & $\begin{array}{c}\text { Treino } \\
\text { semanal }\end{array}$ & $\begin{array}{c}\text { Participação em } \\
\text { competições }\end{array}$ \\
\hline Rendimento máximo & $-0,09$ & $-0,07$ & $-0,08$ & $-0,25$ \\
\hline Ausência de Preocupação & $-0,02$ & $-0,27$ & $-0,31^{*}$ & $-0,04$ \\
\hline Confronto adversidade & $-0,21$ & $-0,20$ & $-0,23$ & $-0,30^{*}$ \\
\hline Concentração & $-0,16$ & $-0,09$ & $-0,06$ & $-0,14$ \\
\hline Formulação de Objetivos & $-0,04$ & $-0,26$ & $-0,28^{*}$ & $-0,05$ \\
\hline Confiança e motivação & $-0,02$ & $-0,24$ & $-0,25$ & $-0,11$ \\
\hline Treinabilidade & $-0,16$ & $-0,11$ & $-0,11$ & $-0,02$ \\
\hline Índice de confronto geral & $-0,05$ & $-0,31^{*}$ & $-0,35^{*}$ & $-0,18$ \\
\hline
\end{tabular}

* Diferença significativa $(p<0,05)$

\section{DISCUSSÃO}

O presente estudo buscou analisar a relação entre as estratégias de coping, estado de estresse e as características de treinamento dos atletas de MMA. Os resultados evidenciaram que os atletas cujo esporte é a principal fonte de renda apresentaram maior estado de estresse, quanto maior a participação em competições, maior o risco para a ocorrência de lesões. Quanto às estratégias de coping, observou-se que os atletas com maior volume de treinamento diário e semanal utilizam estratégias de "ausência de preocupações", "formulação de objetivos" e têm um maior "índice de confronto geral".

O fato de ter no esporte sua principal fonte de renda (Tabela 2) está relacionado a alta ocorrência de estresse dos atletas, uma vez que é esperado que estes sempre alcancem um bom desempenho, devido à sua dedicação exclusiva ao esporte. O elevado estado estresse encontrado pode ocorrer devido a um desequilíbrio entre as exigências da profissão e a utilização de seus recursos pessoais para o enfrentamento de situações estressantes ${ }^{14}$. Isto porque, devido às características da modalidade, o lutador está sujeito a sofrer lesões e ficar muito tempo afastado das lutas, o que pode acarretar perda de patrocínio, gerando insegurança ${ }^{29}$.

Altos níveis de estresse também podem ser causados devido aos déficits de recuperação dos lutadores, ou à interrupção dos seus períodos de descanso (solicitação técnico, colegas de time, competições, entre outros) interferindo de forma negativa no seu desempenho ${ }^{26}$. No entanto, é necessário que o atleta se recupere para que o nível de estresse em seu organismo diminua. A recuperação engloba processos para o restabelecimento dos recursos físicos e psicológicos, que permite a utilização de tais recursos novamente ${ }^{30}$.

A dimensão "autorregulação", que se refere ao uso de habilidades psicológicas, foi maior nos lutadores que estavam no período pré-competitivo. No estudo de Bresciani et al. ${ }^{31}$, verificou-se que com a aproximação do período competitivo os atletas apresentaram maiores níveis no domínio "estar em forma", enquanto a "autorregulação" teve uma redução significante. Contudo, nos estudos de Noce et al. ${ }^{23} \mathrm{e}$ Faude $^{32}$, este domínio não apresentou diferença ao longo dos períodos de descanso e a aproximação da competição. 
A autorregulação, sobretudo no período pré-competitivo, é importante para os lutadores no sentido de estabelecer seus objetivos, avaliam seu progresso e ajustam seu comportamento para que sejam atingidos ${ }^{33}$, controlando suas emoções, disciplinando-se e colaborando nas estratégias de enfrentamento das dificuldades do esporte ${ }^{34}$. Estas características são essenciais para atletas de MMA, isto porque ao se preparar para a luta, os atletas devem estabelecer uma estratégia para vencer seu adversário e controlar suas emoções, bem como ser capaz de utilizar suas habilidades psicológicas a fim de melhorar o desempenho ${ }^{35}$.

A utilização de estratégias de enfrentamento de estresse tem sido descrita como um diferencial entre atletas de sucesso. Recentemente, Jowet e Spray ${ }^{12}$, em seu estudo com atletas britânicos de elite, verificaram que o senso de eficácia, disciplina, competitividade e o uso de estratégias de coping (enfrentamento) foi fator de suma importância em seu desempenho durante a carreira esportiva. Resultados semelhantes foram descritos por Gould et al. ${ }^{13}$, nos quais ex-atletas campeões olímpicos relataram ser mais otimistas, confiantes, esperançosos e utilizarem mais estratégias de coping.

A utilização de estratégias de coping é uma habilidade psicológica importante e auxilia os atletas a responderem às situações estressantes de maneira adequada, gerenciando suas emoções de forma mais eficaz, lidando melhor com as adversidades e controlando melhor suas emoções. Além disso, sua utilização diminui os níveis de ansiedade, fazendo com que o atleta tenha maior sensação de bem-estar, autoconfiança e motivação.

As estratégias de enfrentamento são aliadas dos atletas para que possam melhorar seu desempenho, uma vez que, ao utilizar de forma efetiva as estratégias de coping, estarão preparados para enfrentar situações estressantes com as quais podem se deparar durante competições e treinamento. No entanto, quando um atleta não consegue lidar com o estresse pode ter seu desempenho afetado de forma negativa ${ }^{16}$.

As limitações apresentadas na presente pesquisa foram o fato de ainda não existir um ranking da modalidade, impossibilitando inferência entre os níveis de estresse e desempenho desta modalidade e o número de atletas participantes da pesquisa, uma vez que foram avaliados apenas atletas de um estado. Para futuras pesquisas, sugere-se que sejam feitas comparações das habilidades psicológicas destes atletas em função do ranking. Além disto, sugere-se que sejam realizados acompanhamentos dos níveis de estresse durante o período de treinamento, sobretudo na fase em que é necessário que os atletas percam peso.

\section{CONCLUSÃO}

Conclui-se que os lutadores cuja principal fonte de renda é o MMA apresentam elevado estado de estresse. Lutadores de MMA com um maior volume de treino utilizam uma diversidade de estratégias de coping voltada ao estabelecimento de objetivos no que se refere ao desempenho e um maior confronto com as adversidades.

Como implicações práticas, sugere-se que sejam realizadas avaliações dos níveis de estresse para que os treinadores possam adequar os treinamentos de modo a não sobrecarregarem os atletas; diminuírem a possibilidade de ocorrência de lesões provenientes do treinamento, além das lesões características da modalidade e evitar o aparecimento da síndrome de overtraining e overraching.

Todos os autores declararam não haver qualquer potencial conflito de interesses referente a este artigo.

CONTRIBUIÇÕES DOS AUTORES: Cada autor contribuiu individual e significativamente para o desenvolvimento do manuscrito. IB (0000-0002-2113-8709)* e LCAC (0000-0002-8155-2776)* foram os principais contribuintes na redação do manuscrito. JB (0000-0002-8238-5682)*, PCBP (0000-0002-7817-2860)* e JLLV (0000-0003-04538185)* realizaram a coleta de dados e análise estatística. Todos os autores realizaram a pesquisa bibliográfica e a revisão do manuscrito, contribuindo com o conceito intelectual do estudo. *Número ORCID (Open Researcher and Contributor ID).

\section{REFERÊNCIAS}

1. Amtmann JA. Self-reported training methods of mixed martial artists at a regional reality fighting event. J Strength Cond Res. 2004;18(1):194-6.

2. Bastidas N, Levine JP, Stile FL. The "sweet science" of reducing periorbital lacerations in mixed martial arts. Ann Plast Surg. 2012;68(1):43-5.

3. Massey WV, Meyer BB, Naylor AH. Toward a grounded theory of self-regulation in mixed martial arts. Psychol Sport Exerc. 2013;14(1):12-20

4. Buse GJ. No holds barred sport fighting: a 10 year review of mixed martial arts competition. $\mathrm{Br} J$ Sports Med. 2006;40(2):169-72.

5. Galetta KM, Barrett J, Allen M, Madda F, Delicata D, Tennant AT, et al. The King-Devick test as a determinant of head trauma and concussion in boxers and MMA fighters. Neurology. 2011;76(17):1456-62.

6. Mcgill S. What I have learned from the great athletes. Procedia IUTAM. 2011;2:128-30.

7. del Vecchio FB, Hirata SM, Franchini E. A review of time-motion analysis and combat development in mixed martial arts matches at regional level tournaments. Percept Mot Skills. 2011;112(2):639-48.

8. Amtmann J. Anticipating and training for the worst-case metabolic scenario: a comment on Del Vecchio, Hirata, and Franchini (2011). Percept Mot Skills. 2012;114(1):123-4.

9. Brewer G, Howarth S. Sport, attractiveness and aggression. Pers Indiv Dif. 2012;53(5):640-43

10. Paillard T. Physiological profile of fighters influences training organization in combat sports: response to Del Vecchio, Hirata, and Franchini (2011). Percept Mot Skills. 2011;113(3):803-4.

11. Spencer DC. Habit(us), body techniques and body callusing: an ethnography of mixed martial arts. Body Soc. 2009;14(4):119-43.

12. Jowett N, Spray CM. British Olympic hopefuls: The antecedents and consequences of implicit ability beliefs in elite track and field athletes. Psychol Sport Exerc. 2013;14(2):145-53.

13. Gould D, Dieffenbach K, Moffet A. Psychological characteristics and their development in Olympic champions. J Appl Sport Psychol. 2002;14(3):172-204.

14. Lazarus RS, Folkman, S. Stress, appraisal, and coping. New York: Springer; 1999

15. Calmeiro L, Tenenbaum G, Eccles D. Event-sequence analysis of appraisals and coping during trapshooting perfomance. J Appl Sport Pshycol. 2010;22(4):392-7.

16. Lazarus RS. How emotions influence performance in competitive sports. J Sport Psychol. 2000;14:229-52.

17. Stefanello JMF. Situações de estresse no vôlei de praia de alto rendimento: um estudo de caso com uma dupla olímpica. Rev Port Cien Desp. 2007;7(2):232-44.

18. Jorge SR, Santos PB, Stefanello JMF. O cortisol salivar como resposta fisiológica ao estresse competitivo: uma revisão sistemática. Rev Educ Física/UEM. 2010;21(4):677-86.

19. Simim MAM, Samulski DM, Ferreira RM. Análise do estresse em atletas de futebol para amputados Rev Educ Física/UEM. 2010;21(2):237-44.
20. De Rose D, Deschamps S, Korsakas P. Situaçöes causadoras de stress no basquetebol de alto rendimento: fatores extracompetitivos. Rev Bras Ciên Mov. 2001;9(1):25-30.

21. Brink MS, Visscher C, Arends S, Zwerver J, Post WJ, Lemmink KA. Monitoring stress and recovery: new insights for the prevention of injuries and illnesses in elite youth soccer players. Br J Sports Med. 2010;44(11):809-15

22. Coutts AJ, Reaburn P. Monitoring changes in rugby league players' perceived stress and recovery during intensified training. Percept Mot Skills. 2008;106(3):904-16.

23. Noce F. Análise dos sintomas de overtraining durante os períodos de treinamento e recuperação: um estudo de caso com uma equipe feminina da Superliga de voleibol 2003/2004. Rev Bras Med Esporte. 2011;17(6):397-400.

24. Smith RE, Schutz RW, Smoll FL, Ptacek JT. Development and validation of a multidimensional measure of sportspecific psychological skills: the Athletic Coping Skills Inventory-28. J Sport Exerc Psychol. 1995;17(4):379-98

25. Serpa S, Palmeira AL. ACSI 28: estudo das experiências desportivas. Lisboa: Laboratório de Psicologia do desporto/Faculdade de Motricidade Humana/Universidade Técnica de Lisboa; 1997.

26. Kellmann M, Kallus K, Samulski DM, Costa L, Simola RAP. Questionário de estresse e recuperação para atletas. In: Manual do usuário. Belo Horizonte: Escola de Educação Física, Fisioterapia e Terapia Ocupacional/UFMG; 2009

27. Costa LOP, Samulski DM. Overtraining em atletas de alto nível - Uma revisão literária. Rev Bras Ciên Mov. 2005;13(2):123-34

28. Nunnally JC. Psychometric theory. New York: Mc-Graw-Hill Book; 1978

29. Marques ACP, Rosado AFB. Situações estressantes nos atletas de basquetebol de alta competição. Rev Bras Educ Fís Esporte. 2005;19(1):71-87.

30. Kellmann M, Güntner K. Changes in stress and recovery, in elite rowers during preparation for the olympic games. Med Sci Sports Exerc. 2000;32(3):676-83.

31. Bresciani G, Cuevas MJ, Garatachea N, Molinero O, Almar M, Paz JA et al. Monitoring biological and psychological measures throughout an entire season in male handball players. Europ J Sports Sci. 2010;10(6):377-84.

32. Faude $\mathrm{O}$, Kellmann M, Ammann T, Schnittker R, Meyer T. Seasonal changes in stress indicators in high level football. Int J Sports Med. 2011;32(4):259-65.32

33. Gazzaniga MS, Heatherton TF. Ciência psicológica: mente, cérebro e comportamento. 2a. ed. Porto Alegre: Artmed; 2005.

34. Snyder C, Lopez SJ. Psicologia positiva: uma abordagem científica e prática das qualidades humanas. Porto Alegre: Artmed; 2009.

35. Weinberg R, Gould D. Fundamentos da psicologia aplicada ao exercício e ao esporte. 4a. ed. Porto Alegre: Artmed; 2008 